\title{
Hedging default risks of CDOs in Markovian contagion models
}

\author{
J.-P. Laurent, A. Cousin, J-D. Fermanian ${ }^{1}$
}

10 May 2007

\begin{abstract}
We describe a hedging strategy of CDO tranches based upon dynamic trading of the corresponding credit default swap index. We rely upon a homogeneous Markovian contagion framework, where only single defaults occur. In our framework, a CDO tranche can be perfectly replicated by dynamically trading the credit default swap index and a risk-free asset. Default intensities of the names only depend upon the number of defaults and are calibrated onto an input loss surface. Moreover, numerical implementation can be carried out fairly easily thanks to a recombining tree describing the dynamics of the aggregate loss. Both continuous time market and its discrete approximation are complete. The computed credit deltas can be seen as a credit default hedge and may also be used as a benchmark to be compared with the market credit deltas.
\end{abstract}

Keywords: CDOs, hedging, contagion model, Markov chain, recombining tree.

\section{Introduction}

When dealing with CDO tranches, the market approach to the derivation of credit default swap deltas consists in bumping the credit curves of the names and computing the ratios of changes in present value of the CDO tranches and the hedging credit default swaps. This involves a pricing engine for CDO tranches, usually some mixture of copula and base correlation approaches, leading to so-called sticky deltas. The only rationale of this modus operandi is local hedging with respect to credit spread risks, provided that the trading books are marked-to-market with the same pricing engine. Even when dealing with small changes in credit spreads, there is no guarantee that this would lead to appropriate credit deltas. For instance one can think of changes in base correlation correlated with changes in credit spreads. Moreover, the standard approach is not associated with a replicating theory, thus inducing the possibility of unexplained drifts and time decay effects in the present value of hedged portfolios.

\footnotetext{
1 Jean-Paul Laurent is professor at ISFA Actuarial School, Université Lyon 1 and a scientific consultant for BNP Paribas (laurent.jeanpaul@free.fr or laurent.jeanpaul@univ-lyon1.fr, http://laurent.jeanpaul.free.fr), 50 avenue Tony Garnier, 69007, LYON, FRANCE.

Areski Cousin (areski.cousin@univ-lyon1.fr) is a PhD candidate at ISFA Actuarial School, Université Lyon 1, 50 avenue Tony Garnier, 69007, LYON, FRANCE.

Jean-David Fermanian (jean-david.fermanian@uk.bnpparibas.com) is a senior quantitative analyst within FIRST, Quantitative Credit Derivatives Research at BNP-Paribas, 10 Harewood Avenue, LONDON NW1 6AA.

The authors thank Xavier Burtschell, Marek Musiela, Marek Rutkowski and Antoine Savine for useful discussions. This paper has an academic purpose and may not be related to the way BNP Paribas hedges its credit derivatives books.
} 
Unfortunately, the trading desks cannot rely on a sound theory to determine replicating prices of CDO tranches. This is partly due to the dimensionality issue, partly to the stacking of credit spread and default risks. Laurent (2006) considers the case of multivariate intensities in a conditionally independent framework and shows that for large portfolios where default risks are well diversified, one can concentrate on the hedging of credit spread risks and control the hedging errors. In this approach, the key assumption is the absence of contagion effects which implies that credit spreads of survival names do not jump at default times, or equivalently that defaults are not informative.

In this paper, we take an alternative route, concentrating on contagion effects and default risks and neglecting specific credit spread dynamics. Contagion models were introduced to the credit field by Davis and Lo (2001), Jarrow and Yu (2001) and further studied by Yu (2007). Schönbucher and Schubert (2001) show that copula models exhibit some contagion effects and relate jumps of credit spreads at default times to the partial derivatives of the copula. This is also the framework used by Bielecki, Jeanblanc \& Rutkowski (2007) to address the hedging issue. A similar but somehow more tractable approach has been considered by Frey \& Backhaus (2006), since the latter paper considers some Markovian models of contagion. In a copula model, the contagion effects are computed from the dependence structure of default times, while in contagion models the intensity dynamics are the inputs from which the dependence structure of default times is derived. In both approaches, credit spreads shifts occur only at default times. Thanks to this quite simplistic assumption, and provided that no simultaneous defaults occur, it can be shown that the CDO market is complete, i.e. CDO tranche cash-flows can be fully replicated by dynamically trading individual credit spread swaps.

For the paper to be self-contained, we recall in Section 1 the mathematics behind the perfect replicating strategy. The main tool there is a martingale representation theorem for multivariate point processes. In Section 2, we restrict ourselves to the case of homogeneous portfolios with Markovian intensities which results in a dramatic dimensionality reduction for the (risk-neutral) valuation of CDO tranches and the hedging of such tranches as well. We find out that the aggregate loss is associated with a pure birth process, which is now well documented in the credit literature. In line with several new papers, Section 3 provides some calibration procedures of such contagion models based on quotes of CDO tranches or on a "loss surface". Section 4 details the computation of dynamic hedging strategies, involving the credit default swap index, through a recombining tree on the aggregate loss. We eventually study the dynamic deltas involved in the replication of CDO tranches through a simple case study.

\section{Theoretical framework}

\subsection{Default times}

Throughout the paper, we will consider $n$ obligors and a random vector of default times $\left(\tau_{1}, \ldots, \tau_{n}\right)$ defined on a probabilistic space $(\Omega, A, P)$. We denote by $N_{1}(t)=1_{\left\{\tau_{1} \leq t\right\}}, \ldots$, $N_{n}(t)=1_{\left\{\tau_{n} \leq t\right\}}$ the default indicator processes and by $H_{i, t}=\sigma\left(N_{i}(s), s \leq t\right), \quad i=1, \ldots, n$, $H_{t}={\underset{i=1}{\vee}}_{i} H_{i, t} \cdot\left(H_{t}\right)_{t \in \mathbb{R}^{+}}$is the natural filtration associated with the default times. 
We denote by $\tau^{1}, \ldots, \tau^{n}$ the ordered default times and assume that no simultaneous defaults can occur, i.e. $\tau^{1}<\ldots<\tau^{n}, P-a . s$. This assumption is important with respect to the completeness of the market. As shown below, it allows to dynamically hedge basket default swaps and CDOs with $n$ credit default swaps ${ }^{2}$.

We moreover assume that there exists some $\left(P, H_{t}\right)$ intensities for the counting processes $N_{i}(t), i=1, \ldots, n$, i.e. there exists some (non negative) $H_{t}$-predictable processes $\alpha_{1}^{P}, \ldots, \alpha_{n}^{P}$, such that $t \rightarrow N_{i}(t)-\int_{0}^{t} \alpha_{i}^{P}(s) d s$ are $\left(P, H_{t}\right)$ martingales.

\subsection{Market assumptions}

For the sake of simplicity, let us assume for a while that instantaneous digital default swaps are traded on the names. An instantaneous digital credit default swap on name $i$ traded at $t$, provides a payoff equal to $d N_{i}(t)-\alpha_{i}^{Q}(t) d t$ at $t+d t . d N_{i}(t)$ is the payment on the default leg and $\alpha_{i}^{Q}(t)$ is the (short term) premium on the default swap.

Since we deal with the filtration generated by default times, the credit default swap premiums are deterministic between two default events. Therefore, we restrain ourselves to a market where only default risks occurs and credit spreads themselves are driven by the occurrence of defaults. In our simple setting, there is no specific credit spread risk. This corresponds to the framework of Bielecki, Jeanblanc \& Rutkowski (2007).

For simplicity, we further assume that (continuously compounded) default-free interest rates are constant and equal to $r$. Given some initial investment $V_{0}$ and some $H_{t}$ - predictable processes $\delta_{1}(\bullet), \ldots, \delta_{n}(\bullet)$ associated with some self-financed trading strategy in instantaneous digital credit default swaps, we attain at time $T$ the payoff $V_{0} e^{r T}+\sum_{i=1}^{n} \int_{0}^{T} \delta_{i}(s) e^{r(T-s)}\left(d N_{i}(s)-\alpha_{i}^{Q}(s) d s\right) . \delta_{i}(s)$ is the nominal amount of instantaneous digital credit default swap on name $i$ held at time $s$. This induces a net cash-flow of $\delta_{i}(s) \times\left(d N_{i}(s)-\alpha_{i}^{Q}(s) d s\right)$ at time $s+d s$, which has to be invested in the default-free savings account up to time $T$.

\subsection{Hedging and martingale representation theorem}

From the absence of arbitrage opportunities, $\alpha_{1}^{Q}, \ldots, \alpha_{n}^{Q}$ are non negative $H_{t}$ - predictable processes. From the same reason, $\left\{\alpha_{i}^{Q}(t)>0\right\} \stackrel{P-a . s .}{=}\left\{\alpha_{i}^{P}(t)>0\right\}$. Under mild regularity

\footnotetext{
${ }^{2}$ In the general case where multiple defaults could occur, we have to consider possibly $2^{n}$ states, and we would require non standard credit default swaps with default payments conditionally on all sets of multiple defaults to hedge CDO tranches.
} 
assumptions, there thus exists a probability $Q$ equivalent to $P$ such that, $\alpha_{1}^{Q}, \ldots, \alpha_{n}^{Q}$ are the $\left(Q, H_{t}\right)$ intensities associated with the default times ${ }^{3}$.

Let us consider some $H_{T}$ measurable $Q$ - integrable payoff $M$. Since $M$ depends upon the default indicators of the names up to time $T$, this encompasses the cases of CDO tranches and basket default swaps, provided that recovery rates are deterministic. Thanks to the integral representation theorem of point process martingales (see Brémaud, chapter III), there exists some $H_{t}$ - predictable processes $\theta_{1}, \ldots, \theta_{n}$ such that:

$$
M=E^{Q}[M]+\sum_{i=1}^{n} \int_{0}^{T} \theta_{i}(s)\left(d N_{i}(s)-\alpha_{i}^{Q}(s) d s\right) .
$$

As a consequence, we can replicate $M$ with the initial investment $E^{Q}\left[M e^{-r T}\right]$ and the trading strategy based on instantaneous digital credit default swaps defined by $\delta_{i}(s)=\theta_{i}(s) e^{-r(T-s)}$ for $0 \leq s \leq T$ and $i=1, \ldots, n$. Let us remark that the replication price at time $t$, is provided by $V_{t}=E^{Q}\left[M e^{-r(T-t)} \mid H_{t}\right]$.

While the use of the representation theorem guarantees that, in our framework, any basket default swap can be perfectly hedged with respect to default risks, it does not provide a practical way to construct hedging strategies. As is the case with interest rate or equity derivatives, exhibiting hedging strategies involves some Markovian assumptions.

\section{Homogeneous Markovian contagion models}

\subsection{Intensity specification}

In the contagion approach, one starts from a specification of the (risk-neutral) default intensities $\alpha_{1}^{Q}, \ldots, \alpha_{n}^{Q}$. Generally speaking, the risk-neutral default intensities depend upon the complete history of defaults. More simplistically, it is often assumed that they depend only upon the current credit status, i.e. the default indicators; thus $\alpha_{i}^{Q}(t), i \in\{1, \ldots, n\}$ is a deterministic function of $N_{1}(t), \ldots, N_{n}(t)$. This is the case of the models of Kusuoka (1999), Jarrow \& Yu (2001), Yu (2007), where the intensities are affine functions of the default indicators ${ }^{4}$.

${ }^{3}$ To avoid unnecessary mathematical difficulty, let us assume that the intensities remain $P-a . s$ positive, meaning that there is actually some default risk at any point in time. Let us define the default risk premium processes $\phi_{1}, \ldots, \phi_{n}$ from $\alpha_{i}^{Q}(t)=\alpha_{i}^{P}(t) \times\left(1+\phi_{i}(t)\right), t \in \mathbb{R}^{+}$, $i=1, \ldots, n$. The density of $Q$ with respect to $P$ is such that: $\frac{d Q}{d P}=\varepsilon\left(\sum_{i=1}^{n} \int \phi_{i}(t)\left(d N_{i}(t)-\alpha_{i}^{P}(t) d t\right)\right)$ where $\varepsilon$ denotes the Doleans-Dade exponential.

${ }^{4}$ This Markovian assumption may be questionable, since the contagion effect of a default event may vanish as time goes by. The Hawkes process, that was used in the credit field by Gieseke and Goldberg (2006), Errais et al. (2007), provides such an example of a more complex time dependence. 
Another practical issue is related to name heterogeneity. Modelling all possible interactions amongst names leads to a huge number of contagion parameters and high dimensional problems, thus to numerical issues. For this practical purpose, we will further restrict to homogeneous models, where all the names share the same risk-neutral intensity. This can be viewed as a reasonable assumption for CDO tranches on large indices, although this is obviously an issue with equity tranches for which idiosyncratic risk is an important feature. Since (risk-neutral) default intensities, $\alpha_{1}^{Q}, \ldots, \alpha_{n}^{Q}$ are equal, we will further denote these individual risk-neutral intensities $\alpha_{\text {. }}^{Q}$.

We will further denote by $N(t)=\sum_{i=1}^{n} N_{i}(t)$ the number of defaults at time $t$ within the pool of assets. For simplicity, we will further assume a constant recovery rate equal to $R$. The aggregate fractional loss at time $t$ is given by: $L(t)=(1-R) \frac{N(t)}{n}$. As a consequence of the no simultaneous defaults assumption, the loss intensity is simply the sum of the individual default intensities and is itself only a function of the number of defaults process. We are thus typically in a bottom-up approach, where one starts with the specification of name intensities and thus derives the dynamics of the aggregate loss.

In a homogeneous and Markovian model, both individual and loss intensities depend only upon $N(t)$. Intensities depending upon the number of defaults are related to mean-field approaches (see Frey \& Backhaus (2006)). We will denote by $\lambda(t, N(t))$ the risk-neutral loss intensity which is related to the individual risk-intensities by:

$$
\lambda(t, N(t))=(n-N(t)) \times \alpha^{Q}(t, N(t)) .
$$

As for parametric specifications, we can think of some additive effects, i.e. the pre-default name intensities take the form $\alpha_{\text {. }}^{Q}(t)=\alpha+\beta N(t)$ for some constants $\alpha, \beta$ as in Herbertsson (2007) or multiplicative effects in the spirit of Davis \& Lo (2001), i.e. the pre-default intensities take the form $\alpha^{Q}(t)=\alpha \times \beta^{N(t)}$. Another possibility is a model of the form $\alpha_{.}^{Q}(t)=\alpha(t)+\beta(N(t))$ which splits the time and number of defaults effects. Of course, we could think of an unconstrained model $\alpha_{\cdot}^{Q}(t, N(t))$. This is discussed in van der Voort (2006), Arnsdorf \& Halperin (2007) or Lopatin \& Misirpashaev (2007). Later on, we provide a calibration procedure of such unconstrained intensities onto market inputs such as expected losses on CDO tranches.

\subsection{Risk-neutral pricing}

Let us remark that in a Markovian homogeneous contagion model, the process $N(t)$ is a Markov chain (under the risk-neutral probability $Q$ ), and more precisely a pure birth process since only single defaults can occur. The generator of the chain, $\Lambda(t)$ is quite simple: 


$$
\Lambda(t)=\left(\begin{array}{ccccccc}
-\lambda(t, 0) & \lambda(t, 0) & 0 & 0 & 0 & 0 & 0 \\
0 & -\lambda(t, 1) & \lambda(t, 1) & 0 & & & 0 \\
0 & & \bullet & \bullet & & 0 \\
0 & & & & \bullet & & 0 \\
0 & & & & & \bullet & 0 \\
0 & & & & & -\lambda(t, n-1) & \lambda(t, n-1) \\
0 & 0 & 0 & 0 & 0 & 0 & 0
\end{array}\right)
$$

The transition matrix between dates $t$ and $t^{\prime}$ is given by $Q\left(t, t^{\prime}\right)=\exp \left(\int_{t}^{t^{\prime}} \Lambda(s) d s\right)$. $\{N(t)=n\}$ is an absorbing state. Such ideas have been put in practice by Arnsdorf \& Halperin (2007), de Koch \& Kraft (2007), Herbertsson (2007), Lopatin \& Misirpashaev (2007) and Herbertsson \& Rootzén (2006) for the pricing of basket credit derivatives and also with respect to calibration issues.

As an example of the above approach, let us consider the replication of a European payoff with payment date $T$, such as a "zero-coupon tranchelet", paying $1_{\{N(T)=k\}}$ at time $T$ for some $k \in\{0,1, \ldots, n\}$. Let us denote by $V(t, N(t))=e^{-r(T-t)} Q(N(T)=k \mid N(t))$ the time $t$ replication price and by $V(t, \bullet)$ the price vector whose components are $V(t, 0), V(t, 1), \ldots V(t, n)$ for $0 \leq t \leq T$. We can thus relate the price vector $V(t, \bullet)$ to the terminal payoff, using the transition matrix:

$$
V(t, \bullet)=e^{-r(T-t)} Q(t, T) V(T, \bullet),
$$

where $V(T, N(T))=\delta_{k}(N(T))$.

Since $e^{-r t} \times V(t, N(t))$ is a $\left(Q, H_{t}\right)$ martingale and using Ito-Doeblin's formula, it can be seen that $V$ solves for: $\frac{\partial V(t, N(t))}{\partial t}+\lambda(t, N(t)) \times(V(t, N(t)+1)-V(t, N(t)))=r V(t, N(t))^{5}$.

\subsection{Computation of credit deltas}

A nice feature of homogeneous contagion models is that the credit deltas, i.e. the holdings in the instantaneous defaults swaps are the same for all (the non-defaulted) names, which results in a dramatic dimensionality reduction.

Let us consider a European ${ }^{6}$ type payoff and denote its replication price at time $t, V(t, \bullet)$. In order to compute the credit deltas, let us remark that:

${ }^{5}$ This leads to the system of backward Kolmogorov differential equations:

$$
\frac{\partial V(t, j)}{\partial t}+\lambda(t, j) \times(V(t, j+1)-V(t, j))=r V(t, j), j=0,1, \ldots, n-1,0 \leq t \leq T,
$$

subject to the boundary conditions: $V(T, j)=\delta_{k}(j), j=0,1, \ldots, n$ and, in the example of a zero-coupon tranchelet, to $V(t, n)=\delta_{k}(n) e^{-r(T-t)}$. 


$$
d V(t, N(t))=\frac{\partial V(t, N(t))}{\partial t} d t+(V(t, N(t)+1)-V(t, N(t))) d N(t)
$$

$V(t, N(t)+1)-V(t, N(t))$ is associated with the jump in the price process when a default occurs in the credit portfolio, i.e. $d N(t)=1$. Thanks to the name homogeneity, $d N(t)=\sum_{i=1}^{n-N(t)} d N_{i}(t)^{7}$ and using:

$$
\frac{\partial V(t, N(t))}{\partial t}+\lambda(t, N(t)) \times(V(t, N(t)+1)-V(t, N(t)))=r V(t, N(t)),
$$

we end up with:

$$
d V(t, N(t))=r V(t, N(t)) d t+\sum_{i=1}^{n-N(t)}(V(t, N(t)+1)-V(t, N(t))) \times\left(d N_{i}(t)-\alpha_{.}^{Q}(t, N(t)) d t\right) .
$$

As a consequence the credit deltas with respect to the individual instantaneous default swaps are equal to:

$$
\delta_{i}(t)=e^{-r(T-t)}(V(t, N(t)+1)-V(t, N(t))) \times\left(1-N_{i}(t)\right),
$$

for $0 \leq t \leq T$ and $i=1, \ldots, n$.

Let us recall that $V_{I}(t, N(t))$ denotes the time $t$ price of the equally weighted portfolio involving the defaultable bonds. Let us denote $\delta_{I}(t, N(t))=\frac{V(t, N(t)+1)-V(t, N(t))}{V_{I}(t, N(t)+1)-V_{I}(t, N(t))}$. It can readily be seen that:

$$
d V(t, N(t))=r \times\left(V(t, N(t))-\delta_{I}(t, N(t)) V_{I}(t, N(t))\right) d t+\delta_{I}(t, N(t)) d V_{I}(t, N(t))
$$

As a consequence, we can perfectly hedge a European type payoff, say a zero-coupon CDO tranche, using only the index portfolio and the risk-free asset. The hedge ratio, with respect to the index portfolio is actually equal to $\delta_{I}(t, N(t))=\frac{V(t, N(t)+1)-V(t, N(t))}{V_{I}(t, N(t)+1)-V_{I}(t, N(t))}$. The previous hedging strategy is feasible provided that $V_{I}(t, N(t)+1) \neq V_{I}(t, N(t))$. The usual case corresponds to some positive dependence, thus $\alpha^{Q}(t, 0) \leq \alpha^{Q}(t, 1) \leq \cdots \leq \alpha^{Q}(t, n-1)$. Therefore $V_{I}(t, N(t)+1)<V_{I}(t, N(t))^{8}$. The decrease in the index portfolio value is the consequence of a direct default effect (one name defaults) and an indirect effect related to a positive shift in the credit spreads associated with the non-defaulted names.

\footnotetext{
${ }^{6}$ At this stage, for notational simplicity, we assume that there are no intermediate payments. This corresponds for instance to the case of zero-coupon CDO tranches with up-front premiums. The more general case is considered below.

${ }^{7}$ The last $N(t)$ names have defaulted.

${ }^{8}$ In the case where $\alpha_{.}^{Q}(t, 0)=\alpha_{\cdot}^{Q}(t, 1)=\cdots=\alpha^{Q}(t, n-1)$, there are no contagion effects and default dates are independent. We still have $V_{I}(t, N(t)+1)<V_{I}(t, N(t))$ since $V_{I}(t, k)=e^{-r(T-t)} E^{Q}\left[1-\frac{N(T)}{n} \mid N(t)=k\right]$ is linear in $k$.
} 
The idea of building a hedging strategy based on the change in value at default times was introduced in Arvanitis and Laurent (1999). The rigorous construction of a dynamic hedging strategy in a univariate case can be found in Blanchet-Scalliet and Jeanblanc (2004). Our result can be seen as a natural extension to the multivariate case, provided that we deal with Markovian homogeneous models: we simply need to deal with the number of defaults $N(t)$ and the index portfolio $V_{I}(t, N(t))$ instead of a single default indicator $N_{i}(t)$ and the corresponding defaultable discount bond price.

\subsection{Dynamics of defaultable discount bonds and credit spreads}

We can actually build a bridge between the above Markov chain approach for the aggregate loss and the well known models involving credit migrations. For this purpose, we will derive the dynamics of a (digital) defaultable discount bond associated with name $i \in\{1, \ldots, n\}$ and maturity $T$. The corresponding payoff at time $T$ is equal to $1_{\left\{\tau_{i}>T\right\}}=1-N_{i}(T)$. Let us now consider a portfolio of the previously defined defaultable bonds with holdings equal to $\frac{1}{n}$ for all names. The portfolio payoff is equal to $V_{I}(T, N(T))=1-\frac{N(T)}{n}$. The replication price at time $t$ given that $N(t)=k$ of such a portfolio is equal to $V_{I}(t, k)=e^{-r(T-t)} E^{Q}\left[1-\frac{N(T)}{n} \mid N(t)=k\right]$. Since the names are exchangeable, the $n-k$ non defaulted names have the same price which is thus $\frac{V_{I}(t, k)}{n-k}$. Thus the price time $t$ of the defaultable discount bond, $B_{i}(t, T)$ is given by:

$$
B_{i}(t, T)=\left(1-N_{i}(t)\right) \times \frac{V_{I}(t, N(t))}{n-N(t)}, V_{I}(t, \bullet)=e^{-r(T-t)} Q(t, T) V_{I}(T, \bullet)
$$

where the pre-default intensity of $\tau_{i}$ is equal to $\alpha_{\cdot}^{Q}(t, N(t))=\frac{\lambda(t, N(t))}{n-N(t)}$. When $N(t)=n$, $\alpha_{.}^{Q}(t, N(t))=0$ and $B_{i}(t, T)=0$. Let us remark that the defaultable discount bond price follows a Markov chain with $n+1$ states $\left\{N(t)=0, N_{i}(t)=0\right\}, \ldots,\left\{N(t)=n-1, N_{i}(t)=0\right\}$ and $\left\{N_{i}(t)=1\right\}$. The generator matrix, $\Lambda(t)$, is equal to:

$$
\left(\begin{array}{ccccccc}
-\lambda(t, 0) & ((n-1) / n) \lambda(t, 0) & 0 & 0 & 0 & 0 & \lambda(t, 0) / n \\
0 & -\lambda(t, 1) & ((n-2) /(n-1)) \lambda(t, 1) & 0 & & \lambda(t, 1) /(n-1) \\
0 & 0 & \bullet & \bullet & & \bullet \\
0 & & & & & \bullet \\
0 & & & & & & \bullet \\
0 & & 0 & 0 & 0 & 0 & \lambda(t, n-1) \\
0 & 0 & & & & -\lambda(t, n-1) & \lambda
\end{array}\right)
$$


Thus, the dynamics of the defaultable bond prices can be viewed as a special case of the one studied by Jarrow, Lando and Turnbull (1997) though the economic interpretation of the states slightly differs.

\section{Calibration issues}

As usual, calibration of a model on input prices is a critical issue. We discuss here several procedures to recover loss intensities from a loss surface or more simply from number of default probabilities. Once the loss intensities are computed, we will be able to compute some dynamic credit deltas of CDO tranches. While the pricing and thus the hedging involves a backward procedure, calibration is associated with forward Kolmogorov differential equations ${ }^{9}$.

\subsection{Loss surface and marginal number of defaults probabilities}

Here, for the paper to be self-contained, we recall how the number of defaults distributions can be recovered from the loss-surface which is closely related to the quotes of CDO tranches. The material here is quite standard

We will further denote the marginal number of defaults probabilities by $p(t, k)=Q(N(t)=k)$ for $0 \leq t \leq T, k=0,1, \ldots, n$.

Given a recovery rate of $R$, the (fractional) loss at time $t$ on the credit portfolio is such that $L(t)=(1-R) \frac{N(t)}{n}$. A zero-coupon base correlation tranche with detachment point $\tilde{k}$, $0 \leq \tilde{k} \leq 1$ and maturity $t$ is associated with a payoff equal to: $\min (\tilde{k}, L(t))$. We will denote by $E L(t, \tilde{k})=E^{Q}[\min (\tilde{k}, L(t))]$ the (risk-neutral) expected loss on such a tranche. The mapping $(t, \tilde{k}) \in[0, T] \times[0,1] \rightarrow E L(t, \tilde{k})$ is commonly known as the "loss surface"

We readily get $E L(t, \tilde{k})=\sum_{m=1}^{n} \min \left(\tilde{k}, \frac{m(1-R)}{n}\right) p(t, m)$. Conversely:

$$
p(t, k)=-\frac{n}{1-R} \times\left(E L\left(t, \frac{(k-1) \times(1-R)}{n}\right)-2 E L\left(t, \frac{k \times(1-R)}{n}\right)+E L\left(t, \frac{(k+1) \times(1-R)}{n}\right)\right),
$$

for $k=1, \ldots, n-1$ and $p(t, n)=\frac{n}{1-R} \times\left(E L(t, 1-R)-E L\left(t, \frac{n-1}{n} \times(1-R)\right)\right)$. Eventually, $p(t, 0)$ is obtained from $\sum_{k=0}^{n} p(t, k)=1$. Thus, given a loss surface we can derive the marginal distribution of the number of defaults for any time horizon.

${ }^{9}$ Let us remark that the forward Kolmogorov equations have been used with a different purpose by Schönbucher (2006) (see equation (2.7)). The aim of the previous paper is to construct arbitrage-free, consistent with some complete loss surface, Markovian models of aggregate losses, possibly in incomplete markets, without detailing the feasibility and implementation of replication strategies.

10 See Walker (2006) and Torresetti et al. (2007) for details about the issues at hand. 


\subsection{Calibration equations on a complete loss surface}

We show here a non-parametric fitting procedure of a possibly non time homogeneous pure birth process onto a complete loss surface or equivalently onto the set of marginal distributions of number of defaults ${ }^{11}$. Proceeding heuristically and conditioning on the number of defaults at time $t$, we get:

$$
p(t+d t, k)=p(t, k) \times(1-\lambda(t, k) d t)+p(t, k-1) \times \lambda(t, k-1) d t,
$$

for $k=1, \ldots, n$ and $p(t+d t, 0)=p(t, 0) \times(1-\lambda(t, 0) d t)$, which yields:

$$
\frac{d p(t, k)}{d t}=\lambda(t, k-1) p(t, k-1)-\lambda(t, k) p(t, k) \text {, for } k=1, \ldots, n, \frac{d p(t, 0)}{d t}=-\lambda(t, 0) p(t, 0) .
$$

We refer to Karlin and Taylor (1975) for more details about the forward equations in the case of a pure birth process. The forward equations can be used to compute the loss intensity dynamics $t \in[0, T] \rightarrow \lambda(t, N(t))$, thanks to:

$$
\lambda(t, 0)=-\frac{1}{p(t, 0)} \frac{d p(t, 0)}{d t}, \lambda(t, k)=\frac{1}{p(t, k)}\left[\lambda(t, k-1) p(t, k-1)-\frac{d p(t, k)}{d t}\right] \text { for } k=1, \ldots, n,
$$

Let us remark that we can also write:

$$
\lambda(t, k)=-\frac{1}{p(t, k)} \frac{d \sum_{m=0}^{k} p(t, m)}{d t}=-\frac{1}{Q(N(t)=k)} \frac{d Q(N(t) \leq k)}{d t} .
$$

Let us recall the standard no arbitrage requirements on the marginal number of defaults probabilities: $0 \leq p(t, m) \leq 1, \forall(t, m) \in[0, T] \times\{0,1, \ldots, n-1\}, \sum_{m=0}^{n} p(t, m)=1, \forall t \in[0, T]$ and since $N(t)$ is non decreasing, $\sum_{m=0}^{k} p(t, m) \geq \sum_{m=0}^{k} p\left(t^{\prime}, m\right), \forall k \in\{0,1, \ldots, n\}, \forall t, t^{\prime} \in[0, T]$ and $t \leq t^{\prime}$

Thus, $\lambda(t, k) \geq 0$ thanks to the no arbitrage constraints on the loss surface. Moreover, since $\sum_{m=0}^{n} p(t, m)=1, \frac{d \sum_{m=0}^{n} p(t, m)}{d t}=0$, thus $\lambda(t, n)=0$, i.e. $\{N(t)=n\}$ is absorbing. In other words, the no-arbitrage constraints on the loss surface guarantee the existence of a non-negative loss intensity with the required boundary conditions. Eventually, the name intensities are provided by: $\alpha_{.}^{Q}(t, N(t))=\frac{\lambda(t, N(t))}{n-N(t)}$.

However, despite its simplicity, the previous approach involves some theoretical and practical issues. Firstly, the loss surface may not be consistent with the assumption of no simultaneous defaults. This implies that $\frac{d p(t, m+i)}{d t}=0$ for $t=0, m=0,1, \ldots, n-2$ and $i=2, \ldots, n-m$. A loss surface computed under some arbitrary smoothing procedure is unlikely to fulfil such

\footnotetext{
${ }^{11}$ The calibration idea parallels Dupire (1994), since local dynamics of the underlying process are derived from marginal distributions of options prices thanks to a forward equation.
} 
constraints and will be associated with simultaneous defaults. Though some loss intensity can still be computed, this will not lead to an accurate pricing of CDO tranches, which is actually a failure of the calibration process. Moreover, the computation of expected losses for small time horizons or low detachment points consists in hazardous extrapolations far way from the quotes of liquid tranches. For these reasons, we think that it is more appropriate and reasonable to calibrate the Markov chain of aggregate losses on a discrete set of meaningful expected losses, corresponding to liquid maturities and attachment - detachment points.

\subsection{Calibration on a discrete set of expected losses}

We detail below the process given a single calibration date $T$. For simplicity, we will be given the number of defaults probabilities $p(T, k), k=0,1, \ldots, n$. Clearly, this involves more information that one could directly access through the quotes of liquid CDO tranches, especially with respect to small and large number of defaults, and some kind of interpolation for non standard attachment points is required.

The assumption of time homogeneous loss intensities is the most simple. We further denote by $\lambda_{k}=\lambda(t, k)$ for $0 \leq t \leq T$, the loss intensity for $k=0,1, \ldots, n-1^{12}$. In the case of a discrete set of calibration dates, as in Arnsdorf \& Halperin (2007), we may think of time homogeneous loss intensities between two calibration dates and a bootstrap calibration procedure.

Solving for the forward equations provides $p(T, 0)=e^{-\lambda_{0} T} \quad$ and $p(T, k)=\lambda_{k-1} \int_{0}^{T} e^{-\lambda_{k}(T-s)} p(s, k-1) d s$ for $1 \leq k \leq n-1$. The previous equations can be used to determine $\lambda_{0}, \ldots, \lambda_{n-1}$ iteratively. When $\lambda_{0}, \ldots, \lambda_{n-1}$ are positive and distinct, it can be seen that for any $k \in\{0, \ldots, n-1\}, p(T, k)$ is a decreasing function of $\lambda_{k}$, taking value $\lambda_{k-1} \int_{0}^{T} p(s, k-1) d s$ for $\lambda_{k}=0$ and with a limit equal to zero as $\lambda_{k}$ tends to infinity. As a consequence, there might not exist a solution to the calibration equations ${ }^{13}$ but if there is some solution, it is unique.

When $\lambda_{0}, \ldots, \lambda_{n-1}$ are positive and distinct, we can check that $p(t, k)=\sum_{i=0}^{k} a_{k, i} e^{-\lambda_{i} t}$ for $0 \leq t \leq T$ and $k=0, \ldots, n-1^{14}$. Using the forward equations and starting from $a_{0,0}=1$, we obtain the

${ }^{12}$ Therefore, the pre-default name intensity is such that $\alpha_{.}^{Q}(t, N(t))=\frac{\lambda_{N(t)}}{n-N(t)}$. Let us recall that $\lambda(t, n)=0$.

13 Whenever $p(T, k)>\lambda_{k-1} \int_{0}^{T} p(s, k-1) d s$ for some $k=1, \ldots, n-1$.

${ }^{14}$ Since $\lambda_{n}=0, p(t, n)$ takes a slightly different form. Its detailed expression is useless here since we only need to deal with $p(t, 0), \ldots p(t, n-1)$ to calibrate $\lambda_{0}, \ldots, \lambda_{n-1}$. Let us also 
recurrence equations $a_{k, i}=\frac{\lambda_{k-1}}{\lambda_{k}-\lambda_{i}} a_{k-1, i}$ for $i=0,1, \ldots, k-1, \quad k=1, \ldots, n-1$ and, using $p(0, k)=0, a_{k, k}=-\sum_{i=0}^{k-1} a_{k, i}$. We can compute iteratively $\lambda_{1}, \ldots, \lambda_{n-1}$ by solving the univariate non linear equations: $\sum_{i=0}^{k-1} a_{k-1, i} e^{-\lambda_{i} T} \times\left(\frac{1-e^{-\left(\lambda_{k}-\lambda_{i}\right) T}}{\lambda_{k}-\lambda_{i}}\right)=\frac{p(T, k)}{\lambda_{k-1}}, k=1, \ldots, n-1$. The previous equations have a unique solution provided that: $p(T, k)<\lambda_{k-1} \times\left(\sum_{i=0}^{k-1} a_{k-1, i} \times\left(\frac{1-e^{-\lambda_{i} T}}{\lambda_{i}}\right)\right)$ for $k=1, \ldots, n-1$.

\section{Computation of credit deltas through a recombining tree}

\subsection{Building up a tree}

We now address the computation of CDO tranche deltas with respect to the credit default swap index of the same maturity. As for the hedging instrument, the premium is set at the inception of the deal and remains fixed. Dealing with the credit default swap index at current market conditions would have been another possible choice. The former choice involves the same hedging instrument throughout the trading period. Similarly, we do not take into account roll dates every six months and trade the same index series up to maturity. Switching from one hedging instrument to another could be dealt with very easily in our framework but we thought the computation of the credit deltas with respect to a fixed underlying would be more meaningful.

We recall that the aggregate loss is given by $L(t)=(1-R) \frac{N(t)}{n}$. Let us consider a tranche with attachment point $a$ and detachment point $b, 0 \leq a \leq b \leq 1$. The credit default swap index is simply a [0,1] tranche. We denote by $O(N(t))$ the outstanding nominal on the previous tranche. It is equal to $b-a$ if $L(t)<a$, to $b-a-L(t)$ if $a \leq L(t)<b$ and to 0 if $L(t) \geq b$.

Let us recall that, for a European type payoff the price vector fulfils $V(t, \bullet)=e^{-r\left(t^{\prime}-t\right)} Q\left(t, t^{\prime}\right) V\left(t^{\prime}, \bullet\right)$ for $0 \leq t \leq t^{\prime} \leq T$. The transition matrix can be expressed as $Q\left(t, t^{\prime}\right)=\exp \left(\int_{t}^{t^{\prime}} \Lambda(s) d s\right)$ where $\Lambda(t)$ is the generator matrix associated with the number of defaults process. We will be given a set of node dates $t_{0}=0, \ldots, t_{i}, \ldots, t_{n_{s}}=T$ and denote by $\Delta_{i}=t_{i+1}-t_{i}$ the corresponding time steps. The most simple discrete time approximation one can think of is $Q\left(t_{i}, t_{i+1}\right)=I d+\Lambda\left(t_{i}\right) \times\left(t_{i+1}-t_{i}\right)$. In the time homogeneous framework discussed in the previous section, the generator matrix does not depend on time. We readily

remark that $p(t, n)$ can equally be recovered from $p(t, n)=\lambda_{n-1} \int_{0}^{t} p(s, n-1) d s$ or from $\sum_{k=0}^{n} p(t, k)=1$ 
have $Q\left(N\left(t_{i+1}\right)=k+1 \mid N\left(t_{i}\right)=k\right)=\lambda_{k} \Delta_{i}$ and $Q\left(N\left(t_{i+1}\right)=k \mid N\left(t_{i}\right)=k\right)=1-\lambda_{k} \Delta_{i}$. Thus, the number of defaults process can described through a recombining tree as in van der Voort (2006). One could clearly think of more sophisticated continuous Markov chain techniques, but we think that the tree implementation is quite intuitive from a financial point of view. Convergence of the discrete time Markov chain to its continuous limit is a rather standard issue and will not be detailed here.

Let us denote by $d(i, k)$ the value at time $t_{i}$ when $N\left(t_{i}\right)=k$ of the default payment leg of the CDO tranche ${ }^{15}$. The default payment at time $t_{i+1}$ is equal to $O\left(N\left(t_{i}\right)\right)-O\left(N\left(t_{i+1}\right)\right)$. Thus, $d(i, k)$ is given by the following recurrence equation:

$$
d(i, k)=e^{-r \Delta_{i}}\left(\lambda_{k} \Delta_{i} \times(d(i+1, k+1)+O(k)-O(k+1))+\left(1-\lambda_{k} \Delta_{i}\right) d(i+1, k)\right) .
$$

Let us now deal with a (unitary) premium leg. We denote the regular premium payment dates by $T_{1}, \ldots, T_{p}$ and for simplicity we assume that: $\left\{T_{1}, \ldots, T_{p}\right\} \subset\left\{t_{0}, \ldots, t_{n_{s}}\right\}$. Let us consider some date $t_{i+1}$ and set $l$ such that $T_{l}<t_{i+1} \leq T_{l+1}$. The premium cash-flows can be described as follows: if $t_{i+1}=T_{l+1}$, i.e. $t_{i+1}$ is a regular premium payment date, there is a regular premium cash-flow at time $t_{i+1}$ of $O\left(N\left(T_{l+1}\right)\right) \times\left(T_{l+1}-T_{l}\right)$. Whatever $t_{i+1}$, there is an extra accrued premium payment of $\left(O\left(N\left(t_{i}\right)\right)-O\left(N\left(t_{i+1}\right)\right)\right) \times\left(t_{i+1}-T_{l}\right)$. Thus, if $t_{i+1}$ is a regular premium payment date, the total premium payment is equal to $O\left(N\left(T_{l}\right)\right) \times\left(T_{l+1}-T_{l}\right)$. If $t_{i+1}$ is not a regular premium payment date, we only need to account for a possible accrued premium payment equal to $\left(O\left(N\left(t_{i}\right)\right)-O\left(N\left(t_{i+1}\right)\right)\right) \times\left(t_{i+1}-T_{l}\right)$.

Let us denote by $r(i, k)$ the value at time $t_{i}$ when $N\left(t_{i}\right)=k$ of the unitary premium leg ${ }^{16}$. If $t_{i+1} \in\left\{T_{1}, \ldots, T_{p}\right\}, r(i, k)$ is provided by:

$$
r(i, k)=e^{-r \Delta_{i}}\left(O(k) \times\left(T_{l+1}-T_{l}\right)+\lambda_{k} \Delta_{i} \times(r(i+1, k+1))+\left(1-\lambda_{k} \Delta_{i}\right) r(i+1, k)\right)
$$

If $t_{i+1} \notin\left\{T_{1}, \ldots, T_{p}\right\}$, then:

$$
r(i, k)=e^{-r \Delta_{i}}\left(\lambda_{k} \Delta_{i} \times\left(r(i+1, k+1)+(O(k)-O(k+1)) \times\left(t_{i+1}-T_{l}\right)\right)+\left(1-\lambda_{k} \Delta_{i}\right) r(i+1, k)\right) .
$$

The CDO tranche premium is equal to $s=\frac{d(0,0)}{r(0,0)}$. The value of the CDO tranche (buy protection case) at time $t_{i}$ when $N\left(t_{i}\right)=k$ is given by $V_{C D O}(i, k)=d(i, k)-s r(i, k)$. The equity tranche needs to be dealt with slightly differently since its spread is set to $s=500 \mathrm{bp}$. However, the value of the CDO equity tranche is still given by $d(i, k)-s r(i, k)$.

\footnotetext{
${ }^{15}$ We consider the value of the default leg immediately after $t_{i}$. Thus, we do not consider a possible default payment at $t_{i}$.

${ }^{16}$ As for the default leg, we consider the value of the premium leg immediately after $t_{i}$. Thus, we do not take into account possible premium payments at $t_{i}$ either.
} 
As for the credit default swap index, we will denote by $r_{I S}(i, k)$ and $d_{I S}(i, k)$ the values of the premium and default legs. The credit default swap index spread at time $t_{i}$ when $N\left(t_{i}\right)=k$ is given by $s_{I S}(i, k) \times r_{I S}(i, k)=d_{I S}(i, k)$. The value of the credit default swap index at node $(i, k)$ is given by $V_{I S}(i, k)=d_{I S}(i, k)-s_{I S}(0,0) \times r_{I S}(i, k)$.

As usual in binomial trees, $\delta(i, k)$ is the ratio of the difference of the option value (at time $\left.t_{i+1}\right)$ in the upper state ( $k+1$ defaults) and lower state ( $k$ defaults) and the corresponding difference for the underlying asset. In our case, both the CDO tranche and the credit default swap index are “dividend-baring”. For instance, when the number of defaults switches for $k$ to $k+1$, the default leg of the CDO tranche is associated with a default payment of $O(k)-O(k+1)$. Similarly, given the above discussion, when the number of defaults switches for $k$ to $k+1$, the premium leg of the CDO tranche is associated with an accrued premium payment of $-s \times 1_{t_{i+1} \notin\left\{T_{1}, \ldots, T_{p}\right\}}(O(k)-O(k+1)) \times\left(t_{i+1}-T_{l}\right)$. Thus, when a default occurs the change in value of the $\mathrm{CDO}$ tranche is the outcome of a capital gain of $V_{C D O}(i+1, k+1)-V_{C D O}(i+1, k)$ and of a cash-flow of $(O(k)-O(k+1)) \times\left(1-s \times 1_{t_{i+1} \notin\left\{T_{1}, \ldots, T_{p}\right\}} \times\left(t_{i+1}-T_{l}\right)\right)$. As for the credit default swap index, the change in the outstanding nominal is simply $\frac{1-R}{n}$ where $R$ is the recovery rate and $n$ the number of names.

The credit delta of the CDO tranche at node $(i, k)$ with respect to the credit default swap index is thus given by:

$$
\delta(i, k)=\frac{V_{C D O}(i+1, k+1)-V_{C D O}(i+1, k)+(O(k)-O(k+1)) \times\left(1-s \times 1_{t_{i+1} \notin\left\{T_{1}, \ldots, T_{p}\right\}} \times\left(t_{i+1}-T_{l}\right)\right)}{V_{I S}(i+1, k+1)-V_{I S}(i+1, k)+\frac{1-R}{n} \times\left(1-S_{I S}(0,0) \times 1_{t_{i+1} \notin\left\{T_{1}, \ldots, T_{p}\right\}} \times\left(t_{i+1}-T_{l}\right)\right)} .
$$

Let us remark that using the previous credit deltas leads to a perfect replication of a CDO tranche within the tree, which is feasible since the approximating discrete market is complete.

We also remark that we can easily compute credit deltas with respect to the credit default swap index traded at current market conditions by using $s_{I S}(i, k)$ instead of $s_{I S}(0,0)$ when computing $V_{I S}$ at time $t_{i+1}$ and in the $\delta(i, k)$ expression.

\subsection{Numerical results}

In this numerical illustration, the loss intensities $\lambda_{k}$ are computed from a loss distribution generated from a one factor Gaussian copula. The correlation parameter is equal to $\rho=0.3$, the credit spreads are all equal to 20 basis points per annum, the recovery rate is such that $R=40 \%$ and the maturity is $T=5$ years. The number of names is $n=125$. The continuously compounded default free rate is $r=3 \%$. 
Loss intensities $\lambda_{k}$ are calibrated as previously discussed. Since the default probabilities $p(T, k)$ are insignificant for large number of defaults, we consider that $\lambda_{k}=0$ for $k=n_{\max }, \ldots, n$. Thus, the number of default cannot exceed $n_{\max }=50$ or in probabilistic terms $\left\{n_{\max }=50\right\}$ is absorbing. Table 1 shows the calibrated loss intensities $\lambda_{k}$ for $k=0, \ldots, n_{\max }-1$.

\begin{tabular}{|c|c|c|c|c|c|c|c|c|c|}
\hline $\mathbf{0}$ & $\mathbf{1}$ & $\mathbf{2}$ & $\mathbf{3}$ & $\mathbf{4}$ & $\mathbf{5}$ & $\mathbf{6}$ & $\mathbf{7}$ & $\mathbf{8}$ & $\mathbf{9}$ \\
\hline 0.27 & 0.41 & 0.57 & 0.75 & 0.94 & 1.15 & 1.36 & 1.59 & 1.82 & 2.05 \\
\hline \hline $\mathbf{1 0}$ & $\mathbf{1 1}$ & $\mathbf{1 2}$ & $\mathbf{1 3}$ & $\mathbf{1 4}$ & $\mathbf{1 5}$ & $\mathbf{1 6}$ & $\mathbf{1 7}$ & $\mathbf{1 8}$ & $\mathbf{1 9}$ \\
\hline 2.29 & 2.54 & 2.79 & 3.04 & 3.29 & 3.55 & 3.80 & 4.06 & 4.32 & 4.58 \\
\hline \hline $\mathbf{2 0}$ & $\mathbf{2 1}$ & $\mathbf{2 2}$ & $\mathbf{2 3}$ & $\mathbf{2 4}$ & $\mathbf{2 5}$ & $\mathbf{2 6}$ & $\mathbf{2 7}$ & $\mathbf{2 8}$ & $\mathbf{2 9}$ \\
\hline 4.84 & 5.10 & 5.35 & 5.61 & 5.87 & 6.12 & 6.38 & 6.63 & 6.88 & 7.13 \\
\hline \hline $\mathbf{3 0}$ & $\mathbf{3 1}$ & $\mathbf{3 2}$ & $\mathbf{3 3}$ & $\mathbf{3 4}$ & $\mathbf{3 5}$ & $\mathbf{3 6}$ & $\mathbf{3 7}$ & $\mathbf{3 8}$ & $\mathbf{3 9}$ \\
\hline 7.37 & 7.62 & 7.86 & 8.10 & 8.34 & 8.57 & 8.80 & 9.03 & 9.25 & 9.47 \\
\hline \hline $\mathbf{4 0}$ & $\mathbf{4 1}$ & $\mathbf{4 2}$ & $\mathbf{4 3}$ & $\mathbf{4 4}$ & $\mathbf{4 5}$ & $\mathbf{4 6}$ & $\mathbf{4 7}$ & $\mathbf{4 8}$ & $\mathbf{4 9}$ \\
\hline 9.69 & 9.91 & 10.12 & 10.32 & 10.53 & 10.72 & 10.92 & 11.11 & 11.30 & 11.48 \\
\hline
\end{tabular}

Table 1. $\lambda_{k}, k=0, \ldots, n_{\max }-1$

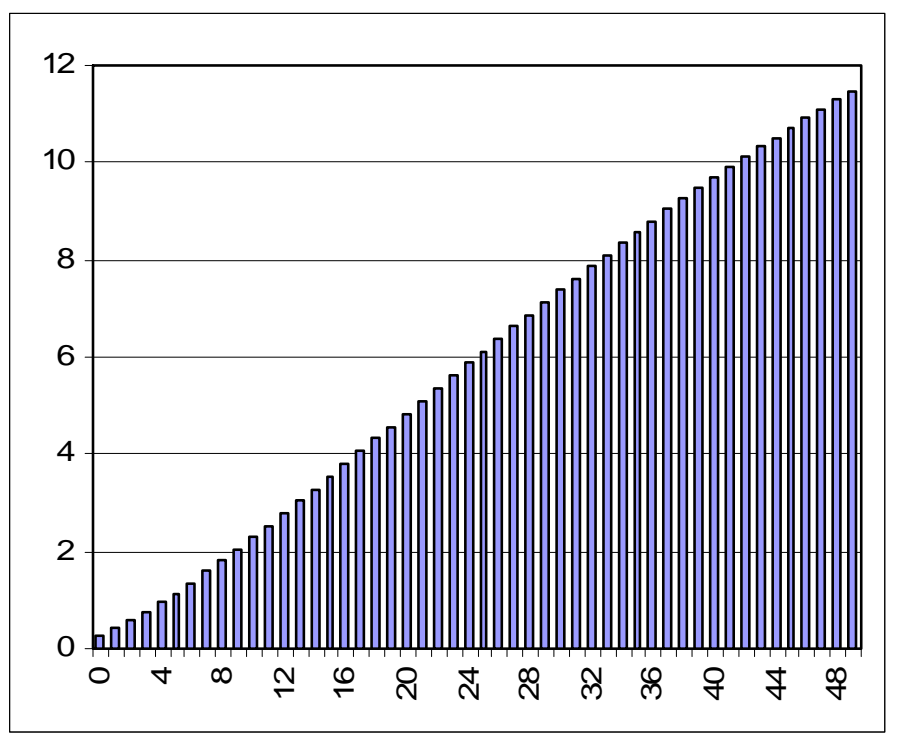

Figure 2. $\lambda_{k}, k=0, \ldots, n_{\max }-1$

As can be seen from Figure 2, the loss intensity $\lambda_{k}$ changes almost linearly with respect to the number of defaults. Table 2 shows the dynamics of the credit default swap index spreads $s_{I S}(i, k)$ along the nodes of the tree. 


\begin{tabular}{|c|c|c|c|c|c|c|c|c|}
\hline & \multicolumn{7}{|c|}{ Weeks } \\
\hline & & 0 & 14 & 28 & 42 & 56 & 70 & 84 \\
\hline \multirow{16}{*}{ 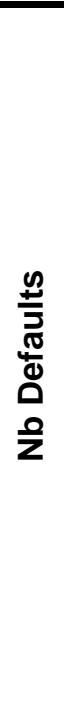 } & 0 & 20 & 19 & 19 & 18 & 18 & 17 & 17 \\
\hline & 1 & 0 & 31 & 30 & 29 & 28 & 27 & 26 \\
\hline & 2 & 0 & 46 & 44 & 43 & 41 & 40 & 38 \\
\hline & 3 & 0 & 63 & 61 & 58 & 56 & 54 & 52 \\
\hline & 4 & 0 & 83 & 79 & 76 & 73 & 70 & 67 \\
\hline & 5 & 0 & 104 & 99 & 95 & 91 & 87 & 83 \\
\hline & 6 & 0 & 127 & 121 & 116 & 111 & 106 & 101 \\
\hline & 7 & 0 & 151 & 144 & 138 & 132 & 126 & 120 \\
\hline & 8 & 0 & 176 & 169 & 161 & 154 & 146 & 140 \\
\hline & 9 & 0 & 203 & 194 & 185 & 176 & 168 & 160 \\
\hline & 10 & 0 & 230 & 219 & 209 & 200 & 190 & 181 \\
\hline & $\overline{11}$ & 0 & 257 & 246 & 235 & 224 & 213 & 203 \\
\hline & 12 & 0 & 284 & 272 & 260 & 248 & 237 & 225 \\
\hline & $\overline{13}$ & 0 & 310 & 298 & 286 & 273 & 260 & 248 \\
\hline & $\overline{14}$ & 0 & 336 & 324 & 311 & 298 & 284 & 271 \\
\hline & $\overline{15}$ & 0 & 0 & 348 & 336 & 323 & 308 & 294 \\
\hline
\end{tabular}

Table 2. $s_{I S}(i, k)$ in basis points per annum

The credit deltas $\delta(i, k)$ have been computed for the[0,3\%], [3,6\%] and [6,9\%] CDO tranches (see Tables 3, 6 and 7).

\begin{tabular}{|c|c|c|c|c|c|c|c|c|c|}
\hline & \multirow{2}{*}{$\begin{array}{l}\text { OutStanding } \\
\text { Nominal }\end{array}$} & \multicolumn{7}{|c|}{ Weeks } \\
\hline & & & $\overline{0}$ & 14 & 28 & 42 & 56 & 70 & 84 \\
\hline \multirow{8}{*}{$\begin{array}{l}\frac{0}{5} \\
\frac{\pi}{\pi} \\
\frac{\pi}{0} \\
\frac{0}{z}\end{array}$} & 0 & $3.00 \%$ & 0.967 & 0.993 & 1.016 & 1.035 & 1.052 & 1.065 & 1.075 \\
\hline & 1 & $2.52 \%$ & $\overline{0}$ & 0.742 & 0.786 & 0.828 & 0.869 & 0.908 & 0.943 \\
\hline & 2 & $2.04 \%$ & 0 & 0.439 & 0.484 & 0.532 & 0.583 & 0.637 & 0.691 \\
\hline & 3 & $1.56 \%$ & $\overline{0}$ & 0.206 & 0.233 & 0.265 & 0.301 & 0.343 & 0.391 \\
\hline & 4 & $1.08 \%$ & $\overline{0}$ & 0.082 & 0.093 & 0.106 & 0.121 & 0.141 & 0.164 \\
\hline & 5 & $0.60 \%$ & 0 & 0.029 & 0.032 & 0.035 & 0.039 & 0.045 & 0.051 \\
\hline & 6 & $0.12 \%$ & $\overline{0}$ & 0.004 & 0.005 & 0.005 & 0.006 & 0.006 & 0.007 \\
\hline & 7 & $0.00 \%$ & 0 & 0 & 0 & $\overline{0}$ & $\overline{0}$ & 0 & 0 \\
\hline
\end{tabular}

Table 3. $\delta(i, k)$ for the $[0,3 \%]$ equity tranche

The credit deltas $\delta(i, k)$ can be decomposed into a default leg delta $\delta_{d}(i, k)$ and a premium leg delta $\delta_{r}(i, k)$ as follows: $\delta(i, k)=\delta_{d}(i, k)-s \delta_{r}(i, k)$ with

$$
\delta_{d}(i, k)=\frac{d(i+1, k+1)-d(i+1, k)+O(k)-O(k+1)}{V_{I S}(i+1, k+1)-V_{I S}(i+1, k)+\frac{1-R}{n} \times\left(1-s_{I S}(0,0) \times 1_{t_{t+1} \notin\left\{T_{1}, \ldots, T_{p}\right\}} \times\left(t_{i+1}-T_{l}\right)\right)}
$$

and

$$
\delta_{r}(i, k)=\frac{r(i+1, k+1)-r(i+1, k)+(O(k)-O(k+1)) 1_{t_{i+1}\left\{\left\{T_{1}, \ldots, T_{p}\right\}\right.} \times\left(t_{i+1}-T_{l}\right)}{V_{I S}(i+1, k+1)-V_{I S}(i+1, k)+\frac{1-R}{n} \times\left(1-S_{I S}(0,0) \times 1_{t_{t+1}\left\{\left\{T_{1}, \ldots, T_{p}\right\}\right.} \times\left(t_{i+1}-T_{l}\right)\right)}
$$




\begin{tabular}{|c|c|c|c|c|c|c|c|c|c|}
\hline & \multirow{2}{*}{$\begin{array}{l}\text { OutStanding } \\
\text { Nominal }\end{array}$} & \multicolumn{7}{|c|}{ Weeks } \\
\hline & & & 0 & 14 & 28 & 42 & 56 & 70 & 84 \\
\hline \multirow{8}{*}{$\begin{array}{l}\frac{0}{5} \\
\frac{\pi}{\pi} \\
\frac{\pi}{0} \\
0 \\
\frac{0}{2}\end{array}$} & 0 & $3.00 \%$ & $\overline{0.814}$ & 0.843 & 0.869 & 0.893 & 0.915 & 0.933 & 0.949 \\
\hline & 1 & $2.52 \%$ & 0 & 0.614 & 0.658 & 0.702 & 0.746 & 0.787 & 0.827 \\
\hline & 2 & $2.04 \%$ & 0 & 0.341 & 0.384 & 0.431 & 0.482 & 0.535 & 0.591 \\
\hline & 3 & $1.56 \%$ & $\overline{0}$ & 0.140 & 0.165 & 0.194 & 0.229 & 0.269 & 0.315 \\
\hline & 4 & $1.08 \%$ & $\overline{0}$ & 0.045 & 0.054 & 0.064 & 0.078 & 0.095 & 0.117 \\
\hline & 5 & $0.60 \%$ & 0 & 0.013 & 0.015 & 0.017 & 0.020 & 0.024 & 0.030 \\
\hline & 6 & $0.12 \%$ & $\overline{0}$ & 0.002 & 0.002 & 0.002 & 0.003 & 0.003 & 0.003 \\
\hline & 7 & $0.00 \%$ & 0 & 0 & 0 & 0 & 0 & 0 & 0 \\
\hline
\end{tabular}

Table 4. $\delta_{d}(i, k)$ for the $[0,3 \%]$ equity tranche

\begin{tabular}{|c|c|c|c|c|c|c|c|c|c|}
\hline & \multirow{2}{*}{$\begin{array}{l}\text { OutStanding } \\
\text { Nominal }\end{array}$} & \multicolumn{7}{|c|}{ Weeks } \\
\hline & & & 0 & 14 & 28 & 42 & 56 & 70 & 84 \\
\hline \multirow{8}{*}{$\begin{array}{l}\frac{n}{5} \\
\frac{\pi}{\pi} \\
\frac{\pi}{0} \\
\text { O0 }\end{array}$} & $\overline{0}$ & $3.00 \%$ & -0.153 & -0.150 & -0.146 & -0.142 & -0.137 & -0.132 & -0.126 \\
\hline & 1 & $2.52 \%$ & $\overline{0}$ & -0.128 & -0.127 & -0.126 & -0.124 & -0.120 & -0.116 \\
\hline & $\overline{2}$ & $2.04 \%$ & $\overline{0}$ & -0.098 & -0.100 & -0.101 & -0.102 & -0.101 & -0.100 \\
\hline & 3 & $1.56 \%$ & $\overline{0}$ & -0.066 & -0.068 & -0.071 & -0.073 & -0.074 & -0.076 \\
\hline & 4 & $1.08 \%$ & $\overline{0}$ & -0.037 & -0.039 & -0.041 & -0.043 & -0.045 & -0.047 \\
\hline & 5 & $0.60 \%$ & $\overline{0}$ & -0.016 & -0.017 & -0.018 & -0.019 & -0.020 & -0.021 \\
\hline & $\overline{6}$ & $0.12 \%$ & 0 & -0.003 & -0.003 & -0.003 & -0.003 & -0.003 & -0.003 \\
\hline & 7 & $0.00 \%$ & $\overline{0}$ & $\overline{0}$ & $\overline{0}$ & $\overline{0}$ & $\overline{0}$ & $\overline{0}$ & $\overline{0}$ \\
\hline
\end{tabular}

Table 5. $s \delta_{r}(i, k)$ for the $[0,3 \%]$ equity tranche

This previous decomposition is useless for the two next tranches [3,6\%] and [6,9\%] since the impact of the CDO tranche premium leg becomes negligible.

\begin{tabular}{|c|c|c|c|c|c|c|c|c|c|}
\hline & \multirow{2}{*}{$\begin{array}{l}\text { OutStanding } \\
\text { Nominal }\end{array}$} & \multicolumn{7}{|c|}{ Weeks } \\
\hline & & & 0 & 14 & 28 & 42 & 56 & 70 & 84 \\
\hline \multirow{14}{*}{ 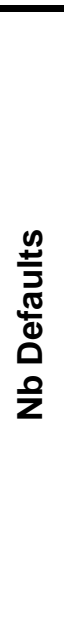 } & 0 & $3.00 \%$ & 0.162 & 0.139 & 0.117 & 0.096 & 0.077 & 0.059 & 0.045 \\
\hline & 1 & $3.00 \%$ & 0 & 0.327 & 0.298 & 0.266 & 0.232 & 0.197 & 0.162 \\
\hline & 2 & $3.00 \%$ & 0 & 0.497 & 0.489 & 0.473 & 0.448 & 0.415 & 0.376 \\
\hline & 3 & $3.00 \%$ & $\overline{0}$ & 0.521 & 0.552 & 0.576 & 0.591 & 0.595 & 0.586 \\
\hline & 4 & $3.00 \%$ & $\overline{0}$ & 0.400 & 0.454 & 0.508 & 0.562 & 0.611 & 0.652 \\
\hline & 5 & $3.00 \%$ & $\overline{0}$ & 0.239 & 0.288 & 0.343 & 0.405 & 0.473 & 0.544 \\
\hline & 6 & $3.00 \%$ & $\overline{0}$ & 0.123 & 0.153 & 0.190 & 0.236 & 0.291 & 0.358 \\
\hline & 7 & $2.64 \%$ & 0 & 0.059 & 0.073 & 0.090 & 0.115 & 0.147 & 0.189 \\
\hline & 8 & $2.16 \%$ & 0 & 0.031 & 0.036 & 0.043 & 0.052 & 0.066 & 0.086 \\
\hline & 9 & $1.68 \%$ & 0 & 0.019 & 0.020 & 0.023 & 0.026 & 0.030 & 0.037 \\
\hline & $\overline{10}$ & $1.20 \%$ & $\overline{0}$ & 0.012 & 0.012 & 0.013 & $\overline{0.014}$ & 0.016 & 0.018 \\
\hline & 11 & $0.72 \%$ & 0 & 0.007 & 0.007 & 0.007 & 0.007 & 0.008 & 0.009 \\
\hline & 12 & $0.24 \%$ & $\overline{0}$ & 0.002 & 0.002 & 0.002 & 0.002 & 0.002 & 0.003 \\
\hline & $\overline{13}$ & $0.00 \%$ & 0 & 0 & 0 & 0 & 0 & 0 & 0 \\
\hline
\end{tabular}

Table 6. $\delta(i, k)$ for the $[3,6 \%]$ tranche 


\begin{tabular}{|c|c|c|c|c|c|c|c|c|c|}
\hline & \multirow{2}{*}{$\begin{array}{c}\text { OutStanding } \\
\text { Nominal }\end{array}$} & \multicolumn{7}{|c|}{ Weeks } \\
\hline & & & 0 & 14 & 28 & 42 & 56 & 70 & 84 \\
\hline \multirow{20}{*}{$\begin{array}{l}\frac{n}{2} \\
\frac{\pi}{\pi} \\
\frac{\pi}{0} \\
\frac{0}{z}\end{array}$} & $\overline{0}$ & $3.00 \%$ & 0.017 & 0.012 & 0.008 & 0.005 & 0.003 & 0.002 & 0.001 \\
\hline & 1 & $3.00 \%$ & 0 & 0.048 & 0.036 & 0.025 & 0.017 & 0.011 & 0.006 \\
\hline & 2 & $3.00 \%$ & 0 & 0.133 & 0.107 & 0.083 & 0.061 & 0.043 & 0.029 \\
\hline & 3 & $3.00 \%$ & $\overline{0}$ & 0.259 & 0.227 & 0.193 & 0.157 & 0.122 & 0.090 \\
\hline & 4 & $3.00 \%$ & $\overline{0}$ & 0.371 & 0.356 & 0.330 & 0.295 & 0.253 & 0.206 \\
\hline & 5 & $3.00 \%$ & $\overline{0}$ & 0.405 & 0.423 & 0.428 & 0.420 & 0.396 & 0.358 \\
\hline & 6 & $3.00 \%$ & 0 & 0.346 & 0.392 & 0.433 & 0.465 & 0.482 & 0.481 \\
\hline & 7 & $3.00 \%$ & 0 & 0.239 & 0.292 & 0.350 & 0.409 & 0.465 & 0.510 \\
\hline & 8 & $3.00 \%$ & 0 & 0.139 & 0.181 & 0.232 & 0.293 & 0.363 & 0.436 \\
\hline & 9 & $3.00 \%$ & $\overline{0}$ & 0.074 & 0.098 & 0.132 & 0.177 & 0.235 & 0.307 \\
\hline & 10 & $3.00 \%$ & $\overline{0}$ & 0.042 & 0.053 & 0.070 & 0.095 & 0.132 & 0.183 \\
\hline & 11 & $3.00 \%$ & 0 & 0.029 & 0.033 & 0.040 & 0.051 & 0.070 & 0.098 \\
\hline & 12 & $3.00 \%$ & 0 & 0.025 & 0.026 & 0.028 & 0.033 & 0.040 & 0.053 \\
\hline & 13 & $2.76 \%$ & $\overline{0}$ & 0.022 & 0.022 & 0.022 & $\overline{0.024}$ & 0.026 & 0.031 \\
\hline & 14 & $2.28 \%$ & $\overline{0}$ & $\overline{0.020}$ & 0.018 & 0.018 & $\overline{0.018}$ & 0.019 & 0.020 \\
\hline & 15 & $1.80 \%$ & 0 & 0 & 0.015 & 0.014 & 0.014 & 0.014 & 0.014 \\
\hline & 16 & $1.32 \%$ & 0 & 0 & 0.013 & 0.011 & $\overline{0.010}$ & 0.010 & 0.010 \\
\hline & 17 & $0.84 \%$ & 0 & 0 & 0.009 & 0.008 & 0.007 & 0.006 & 0.006 \\
\hline & 18 & $0.36 \%$ & 0 & 0 & 0.005 & 0.004 & 0.003 & 0.003 & 0.003 \\
\hline & 19 & $0.00 \%$ & $\overline{0}$ & $\overline{0}$ & 0 & 0 & 0 & $\overline{0}$ & 0 \\
\hline
\end{tabular}

Table 7. $\delta(i, k)$ for the $[6,9 \%]$ tranche

\section{Conclusion}

This paper does not aim at providing a definitive answer to the thorny issue of hedging CDO tranches. For this purpose, we would also need to tackle name heterogeneity, possible non Markovian effects in the dynamics of credit spreads, non deterministic intensities between two default dates, the occurrence of multiple defaults, ... A fully comprehensive approach to the hedging of CDO tranches is likely to be quite cumbersome both on economic and numerical grounds.

The lack of internally consistent methods to hedging CDO tranches has paved the way to a variety of local hedging approaches that do not guarantee the full replication of tranche payoffs. Such incompleteness of the market may not look as such a practical issue as far as trade margins are high and holding periods short. However, we think that there might be a growing concern from investment banks about the long term credit risk management of trading books as the market matures.

A homogeneous Markovian contagion model can be implemented as a recombining binomial tree and thus provides a strikingly easy way to compute dynamic replicating strategies of CDO tranches. While such models have recently been considered for the pricing of exotic basket credit derivatives, our main concern here is to provide a rigorous framework to the hedging issue. From a practical perspective, the assessment of such hedging strategies it is yet unclear. One would obviously think of hedging with respect to default risks only and not with respect to day to day changes in credit spreads. Meanwhile, we think that our approach might be useful to assess the default exposure of CDO tranches by quantifying the credit contagion effects in a reasonable way. 


\section{References}

Arnsdorf, M., and Halperin, I., 2007, BSLP: Markovian bivariate spread-loss model for portfolio credit derivatives, working paper, JP Morgan.

Arvanitis, A., and Laurent, J-P., 1999, On the edge of completeness, RISK, October, 61-65. Bielecki, T., Jeanblanc, M., \& M. Rutkowski, 2007, Hedging of basket credit derivatives in default swap market, Journal of Credit Risk, 3(1).

Blanchet-Scalliet, C., Jeanblanc, M., 2004, Hazard rate for credit risk and hedging defaultable contingent claims, Finance and Stochastics, 8, 145-159.

Brémaud, P., 1981, Point processes and queues: martingale dynamics, Springer-Verlag.

Davis, M., and Lo, V., 2001, Infectious defaults, Quantitative Finance, 1, 382-387.

De Koch, J., and Kraft, H., 2007, CDOs in chains, working paper, University of Kaiserslautern.

Dupire, B., 1994, Pricing with a smile, RISK, January, 18-20.

Frey, R., and Backhaus, J., 2006, Credit derivatives in models with interacting default intensities: a Markovian approach, working paper, University of Leipzig.

Errais, E., Giesecke, K., and L.R. Goldberg, 2007, Pricing credit from the top down with affine point processes, working paper, Stanford university.

Giesecke, K., and L.R. Goldberg, 2006, A top down approach to multi-name credit, working paper, Stanford University.

Herbertsson, A., 2007, Pricing synthetic CDO tranches in a model with default contagion using the matrix-analytic approach, working paper, Göteborg University.

Herbertsson, A., and Rootzén, H., 2006, Pricing k-th to default swaps under default contagion, the matrix-analytic approach, working paper, Göteborg University.

Jarrow, R.A. and Yu, F., 2001, Counterparty risk and the pricing of defaultable securities, Journal of Finance, 53, 2225-2243.

Jarrow, R.A., Lando, D. and S.M. Turnbull, 1997, A Markov model for the term structure of credit risk spreads. Review of Financial Studies, 10(2), 481-523.

Karlin, S. and Taylor H.M., 1975, A First course in stochastic processes, Second Edition, Academic Press.

Kusuoka, S., 1999, A remark on default risk models, Advances in mathematical economics, Vol. 1, 69-82.

Laurent, J-P., 2006, A note on the risk management of CDOs, working paper, ISFA actuarial School, University of Lyon \& BNP Paribas.

Lopatin, A.V. \& Misirpashaev, T., 2007, Two-dimensional Markovian model for dynamics of aggregate credit loss, working paper, NumeriX.

Schönbucher, P.J., 2006, Portfolio losses and the term-structure of loss transition rates: a new methodology for the pricing of portfolio credit derivatives, working paper, ETH Zürich.

Schönbucher, P.J., and Schubert, D., 2001, Copula-dependent default risk in intensity models, working paper, department of statistics, Bonn university.

Torresetti, R., Brigo, D., and Pallavicini, A., 2007, Implied Expected Tranched Loss Surface from CDO Data, working paper, Banca IMI.

van der Voort, M., 2006, An implied loss model, working paper, ABN Amro and Erasmus University.

Walker, M.B., 2007, CDO valuation: term structure, tranche structure, and loss distributions, working paper, university of Toronto.

$\mathrm{Yu}, \mathrm{F} ., 2007$, Correlated defaults in intensity-based models, Mathematical Finance, Vol. 17(2), 155-173. 